\title{
Impacto da mamografia de rastreio na identificação de preditores do câncer de mama no Estado do Tocantins
}

\author{
Impact of screening mammography on the identification of predictors of breast cancer in the State \\ of Tocantins
}

Impacto de la mamografía de detección en la identificación de predictores de cáncer de mama en el Estado de Tocantins

Gisele de Jesus Batista

ORCID: https://orcid.org/0000-0001-9832-3307

Universidade de Gurupi, Brasil

E-mail: gysabatysta@gmail.com

Gabriela Gomes Barros

ORCID: https://orcid.org/0000-0003-0459-6924 Universidade de Gurupi, Brasil

E-mail: gomesbarrosgabriela@gmail.com

Rafaella Antunes Fiorotto de Abreu

ORCID: https://orcid.org/0000-0001-7206-7456 Universidade de Gurupi, Brasil E-mail: rafaella.fiorotto@hotmail.com Julia Sepulvida P. Mota

ORCID: https://orcid.org/0000-0002-7328-8848 Universidade de Gurupi, Brasil E-mail: jspmota@gmail.com

Isana de Oliveira Caetano ORCID: https://orcid.org/0000-0002-9559-9030 Universidade de Gurupi, Brasil E-mail: isanaoc@gmail.com

Geovanna Siqueira Santana ORCID: https://orcid.org/0000-0001-8463-3048 Universidade de Gurupi, Brasil

E-mail: geovanna.siqueira1@gmail.com

Mariana Rodrigues Miranda

ORCID: https://orcid.org/0000-0001-7940-585X

Universidade de Rio Verde, Brasil

E-mail: mariimiranda000@gmail.com

Ana Clara Fernandes Barroso

ORCID: https://orcid.org/0000-0001-5234-4698 Universidade de Gurupi, Brasil

E-mail: anaclarafbarroso1@gmail.com

Isabella Heloiza Santana da Silva ORCID: https://orcid.org/0000-0002-6618-7010 Faculdade Morgana Potrich, Brasil E-mail: isa_heloiza@outlook.com

Isabela Nunes Tavares

ORCID: https://orcid.org/0000-0002-6339-6220

Universidade de Rio Verde, Brasil

E-mail: isabelatavares712@gmail.com

Ana Claudia Saraiva Rocha

ORCID: https://orcid.org/0000-0001-7127-5907 Universidade de Gurupi, Brasil

E-mail: anaclaudiasrocha@hotmail.com

\begin{abstract}
Resumo
Justificativa e Objetivo: No estado do Tocantins, estima-se 290 novos casos de câncer de mama em 2020. De modo que, a mamografia é o principal exame para o rastreio e diagnóstico do câncer de mama. O objetivo deste estudo foi avaliar a proporção da mamografia de rastreio em relação à de diagnóstico frente a identificação de achados preditores do câncer de mama no Tocantins. Métodos: Trata-se de uma pesquisa descritiva analítica, com abordagem quantitativa, em que se realizou busca de dados no Sistema de Informação do Câncer referente aos anos 2015 a 2019. Resultados: Ao serem aplicados testes estatísticos revelou-se que houve evidência suficiente ao nível de significância
\end{abstract}


de 5\% de que há uma associação entre o resultado das mamografias rastreio e diagnósticas e laudos categorizados pelo BI-RADS. Houve maior prevalência de mamografias laudadas como categoria zero. Houveram mais achados de malignidade no grupo de mamografia diagnóstica. Conclusões: A partir do presente estudo pode-se perceber o quanto a mamografia de rastreio foi importante na identificação de alterações (benignas e malignas). Ainda assim, houve maior prevalência de preditivos de malignidade na mamografia de diagnóstico. Deve-se ressaltar que são necessárias ações de rastreamento e diagnóstico precoce conforme as recomendações do Ministério da Saúde, bem como aperfeiçoamento do sistema de laudos, para não haver comprometimento de efetividade do rastreio de câncer de mama.

Palavras-chave: Neoplasias da mama; Mamografia; Diagnóstico precoce; Avaliação em saúde; Programas de rastreamento.

\begin{abstract}
Justification and Objective: In the state of Tocantins, an estimated 290 new cases of breast cancer are expected in 2020. Therefore, mammography is the main exam for the screening and diagnosis of breast cancer. The aim of this study was to evaluate the proportion of screening mammography in relation to that of diagnosis in view of the identification of predictive findings for breast cancer in Tocantins. Methods: This is a descriptive analytical research, with a quantitative approach, in which data were searched in the Cancer Information System for the years 2015 to 2019. Results: When statistical tests were applied, it was revealed that there was sufficient evidence at the time level of significance of 5\% that there is an association between the results of screening mammograms and diagnoses and reports categorized by BI-RADS. There was a higher prevalence of mammograms reported as category zero. There were more malignancy findings in the diagnostic mammography group. Conclusions: From the present study, it can be seen how important screening mammography was in identifying changes (benign and malignant). Even so, there was a higher prevalence of predictors of malignancy on diagnostic mammography. It should be noted that screening and early diagnosis actions are necessary according to the recommendations of the Ministry of Health, as well as improving the system of reports, so that there is no compromise in the effectiveness of breast cancer screening.
\end{abstract}

Keywords: Breast Cancer; Mammography; Early diagnosis; Health evaluation; Screening programs.

\title{
Resumen
}

Justificación y objetivo: En el estado de Tocantins, se estima que hay 290 nuevos casos de cáncer de mama en 2020. Por lo tanto, la mamografía es el principal examen para el cribado y diagnóstico del cáncer de mama. El objetivo de este estudio fue evaluar la proporción de mamografía de cribado en relación con la mamografía de diagnóstico en vista de la identificación de hallazgos que predicen el cáncer de mama en Tocantins. Métodos: Se trata de una investigación analítica descriptiva, con enfoque cuantitativo, en la que se realizó una búsqueda de datos en el Sistema de Información del Cáncer para los años 2015 a 2019.Resultados: Cuando se aplicaron pruebas estadísticas, se reveló que había suficiente Hay evidencia en el nivel de significancia temporal del 5\% de que existe una asociación entre los resultados de las mamografías y los diagnósticos e informes categorizados por BI-RADS. Hubo una mayor prevalencia de mamografías notificadas como categoría cero. Hubo más hallazgos de malignidad en el grupo de mamografía diagnóstica Conclusiones: A partir del presente estudio, se puede ver cuán importante fue la mamografía de detección en la identificación de cambios (benignos y malignos). Aun así, hubo una mayor prevalencia de predictores de malignidad en la mamografía diagnóstica. Cabe destacar que las acciones de cribado y diagnóstico precoz son necesarias según las recomendaciones del Ministerio de Salud, así como mejorar el sistema de informes, para que no se comprometa la efectividad del cribado del cáncer de mama.

Palabras clave: Neoplasias de la mama; Mamografía; Diagnostico temprano; Evaluación de salud; Programas de seguimiento.

\section{Introdução}

O câncer configura como um dos mais importantes problemas de saúde pública no mundo, além de ser um dos quatro responsáveis por morte antes dos 70 anos de idade em um grande número de países. Em 2018, ocorreram no mundo mais de 18 milhões de novos casos, sendo que, o câncer de mama foi o segundo tipo mais incidente no Brasil e no mundo, com cerca de 2,1 milhões de casos, além de ser a neoplasia maligna de maior incidência em mulheres. (Brasil, 2019)

No Brasil, a estimativa de incidência de câncer de mama para o ano de 2020 são de 66.280 novos casos, com uma taxa bruta de 61,61 casos por cada 100.000 mulheres, estimando-se para o estado do Tocantins, 290 novos casos, para cada ano do triênio 2020-2022, com uma taxa bruta de 36,64. (Brasil, 2019)

O câncer de mama é uma neoplasia maligna, de natureza multifatorial, sendo que o tipo histológico mais comum em mulheres é o carcinoma de células epiteliais, que se divide em lesões in situ e invasoras ${ }^{(2,3)}$. Os principais sintomas são nódulos 
na mama e/ou axila, dor mamaria e alterações da pele sobre a mama (abaulamentos ou retrações com aspecto de casca de laranja). Localizam-se principalmente no quadrante superior externo. Na grande parte das vezes as lesões são consideradas indolores, fixas e com bordas irregulares. (Brasil,2019)

A mamografia é uma radiografia da mama, que detecta lesões iniciais. Estudos mostram que a mamografia reduziu em média 30\% dos óbitos de câncer em mulheres com mais de 50 anos. (Brasil, 2006)

Este exame é indicado para mulheres assintomáticas, para rastreamento da neoplasia; e para mulheres sintomáticas, para achados clínicos suspeitos de câncer mamário. (Godinho, Koch, 2002)

Há vários tipos histológicos de moleculares de carcinoma de mama. O tipo histológico invasor mais habitual é o carcionama ductalinfiltrante não especifico (70 a 80\% dos tumores de mama), seguido do carcionoma lobular infiltrante (15\%). As opções mais favoráveis para o tratamento locorregional do câncer de mama são cirurgia e radioterapia, já para o sistêmico são quimioterapia, hormonioterapia e a imunoterapia. (Brasil, 2019)

Diferentes fatores estão associados ao risco de desenvolvimento do câncer de mama. Entre os motivos do aparecimento do câncer de mama destaca-se a idade, principalmente acima dos 50 anos; hábitos de vida ruins como a má alimentação levando à obesidade, alcoolismo e tabagismo; fatores endócrinos e reprodutivos como menarca precoce, menopausa tardia, $1^{\circ}$ gravidez após 30 anos; e por fim, fatores hereditários e genéticos. (WORD, 2018) (Brasil, 2019)

Entre os métodos para identificação de preditores de câncer de mama, nos exames de imagem, está a mamografia, a qual tem a função de detectar precocemente o CA de mama. Se o CA de mama for diagnosticado inicialmente têm possibilidade de recuperação de 95\% e também há menor índice de cirurgias desnecessárias. (Brasil, 2015) (WHO, 2007)

A mamografia é um exame que aborda todas as estruturas habituais da mama, e é por meio desta que se diagnostica o câncer de mama. Há dois tipos relevantes de mamografia, a diagnóstica e a de rastreio. A diagnóstica é aquela em que é motivada por alguma suspeita, seja ela anatômica ou fisiológica, já a de rastreio é aquela em que é feita anualmente, classificada como um exame de rotina, ou seja, as mulheres não possuem nenhuma queixa, mas a fazem com intuito de prevenção. (Brasil, 2015) (Nascimento, Pitta, Rêgo, 2015)

Independente das particularidades do contexto, as ações de detecção precoce do câncer de mama devem ser baseadas nas melhores evidências disponíveis para que sejam efetivas. Diretrizes baseadas em evidências científicas têm sido o instrumento recomendado em todo o mundo para alcançar esses objetivos. (Brasil, 2015)

Os profissionais de saúde devem considerar que uma boa prática em saúde não deve se basear, exclusivamente, na evidência científica. $\mathrm{O}$ uso judicioso das evidências deve considerar outros valores, como a experiência profissional e os valores da paciente. Existem várias questões a serem ponderadas no processo de tomada de decisão em busca do maior benefício e qualidade de vida. (Sacket, 2003)

Aliado às estratégias de prevenção, o CA de mama possui maior chance de cura e aumento de sobrevida, uma vez que possibilita a intervenção antes do desenvolvimento do câncer propriamente dito ou em suas fases iniciais, quando o tratamento é bem mais efetivo. Por esse motivo, em consenso com as diretrizes mais recentes para a detecção precoce de câncer de mama, o Ministério da Saúde indica a necessidade de mulheres de acima de 50 anos fazerem a mamografia. Anteriormente, a recomendação de mamografia era mediante exames físicos anormais ou anualmente a partir dos 40 anos. (Brasil, 2015) (Brasil, 2013) (Migowski, Stein, Ferreira, Nadanovsky, 2018)

Este trabalho tem como objetivo, avaliar a proporção da mamografia de rastreio em relação à de diagnóstico frente a identificação de achados preditores do câncer de mama no Tocantins.

As consequências do atraso no início de tratamento do câncer de mama indicam uma possibilidade de cura diminuída, menor sobrevida, maior morbidade e o aumento do custo de tratamento para o estado, resultando em crescimento da 
mortalidade e maior número de pacientes incapacitados e com sequelas graves após o tratamento, diminuindo assim a qualidade de vida dessa mulher. (Migowski, Stein, Ferreira, Nadanovsky, 2018) (Ferlay,et al. 2012)

Segundo o Projeto Diretrizes, da Associação Médica Brasileira e Conselho Federal de Medicina, que procurou englobar informações da área médica sobre o rastreamento mamográfico, acrescentou na recomendação do Inca que mulheres entre 40 e 49 anos façam anualmente a mamografia, e nas mulheres acima dos 70 anos a decisão deverá ser individual, considerando a expectativa de vida de cada mulher, pois a incidência do câncer de mama aumenta com a idade. (Kemp, Petti, Ferraro, Elias, 2002)

A classificação radiológica inserida no Departamento de Informática do Sistema Único de Saúde (DATASUS) segue a categorização proposta no Breast Imaging Reporting And Data System (BI-RADS®), publicado pelo Colégio Americano de Radiologia (ACR) e traduzido para o Brasil pelo Colégio Brasileiro de Radiologia e Diagnóstico por Imagem (CBR). (RODRIGUES, et.al.2013). É atualmente adotada pelo Sistema Único de Saúde, como forma de qualificar os achados imaginológicos. (Nascimento, Pitta, Rêgo, 2015)

Diante das pesquisas realizadas, levantou-se a problemática que levou ao desenvolvimento desta pesquisa: Seria a mamografia um método eficiente de rastreio para o diagnóstico de câncer de mama?

\section{Metodologia}

O presente estudo desenvolveu-se por meio de pesquisa com abordagem quantitativa, de caráter explicativo, onde foi realizado uma análise documental numa fonte limitada de coleta de informações (DATASUS/SISCAN). A população desse estudo foram todos os pacientes que realizaram mamografia de rastreio, no Brasil, entre os anos de 2015 e 2019 e contabilizados no banco de dados do DATASUS.

Nosso desenho metodológico consistiu em um estudo exploratório sob uma perspectiva qualitativa. Em termos de pesquisa exploratória, nos baseamos na conceituação de Tobar e Yalour (2001), como sendo: “(...) aquela realizada em áreas e sobre problemas dos quais há escasso ou nenhum conhecimento acumulado e sistematizado. Pela natureza de sondagem, não parte de hipóteses. Estas poderão surgir como produto final da pesquisa" (pg 69).

O presente estudo traz uma abordagem qualitativa que busca entender o significado que os sujeitos atribuem às situações vividas e a relação destes significados com a realidade na qual estão inseridos, empregando-a no sentido de uma abordagem voltada para a compreensão de significados, valores culturais e crenças (Minayo, 1999).

Para obtenção desta análise foram empregados os questionamentos: número de pacientes que realizou mamografia de rastreio e mamografia diagnóstica, classificação dentro do parâmetro BI-RADS para resultado de exame de mamografia, faixa etária dos pacientes que realizaram mamografia, presença de risco elevado para câncer de mama como evidência prévia na realização de mamografia.

A existência de associações entre duas variáveis categóricas foi verificada utilizando-se o teste de Qui-Quadrado. Em se verificando diferenças na distribuição, foi utilizado o resíduo ajustado padronizado para identificar as diferenças locais (caselas) com valores absolutos acima de 1,96 indicando evidências de associações (locais) entre as categorias relativas a essas caselas. As comparações de médias entre dois grupos foram realizadas utilizando-se o teste t de Student para amostras independentes. Para todos os testes estatísticos foram utilizados um nível de significância de 5\%.

Para o contexto do referencial teorico, Martins (2001) a pesquisa bibliográfica procura explicar e discutir um tema com base em referências teóricas publicadas em livros, revistas, periódicos e outros. Buscam também, conhecer e analisar conteúdos científicos sobre determinados tema. 


\section{Resultados e Discussão}

É notável a importância de aplicação de métodos de detecção precoce para o câncer de mama e assim otimizar o tratamento. Sob a perspectiva das atuais políticas de saúde buscarem oferecer realização de exames para o rastreamento do câncer, buscou-se analisar o impacto da realização mamografia de rastreio na identificação de achados preditores do câncer de mama no estado do Tocantins.

A categorização do BI-RADS é atualmente, aplicada nos exames de mamografia, ultrassonografia mamária e ressonância nuclear magnética das mamas de forma a assegurar uma maior confiabilidade destes e tornar a sua leitura facilmente interpretada por profissionais do mundo inteiro. Tal método abrange análises de cistos, nódulos e calcificações presentes na mama examinada e visa orientar a conduta médica ante os achados mamográficos podendo ser estes negativos, benignos, provavelmente benignos, suspeitos e altamente suspeitos como vemos na (Figura 1). (American, 2016) (Brasil, 2013)

Figura 1. Classificação BI-RADS® para laudo mamográfico e risco de câncer.

\begin{tabular}{ll}
\hline Categoria BI-RADS & Achados mamográficos \\
\hline $\mathbf{1}$ & Sem achados \\
\hline $\mathbf{2}$ & Achados benignos \\
\hline $\mathbf{3}$ & Achados provavelmente benignos \\
\hline $\mathbf{4}$ & Achados suspeitos de malignidade \\
\hline $\mathbf{5}$ & Achados altamente suspeitos de malignidade \\
\hline $\mathbf{6}$ & Diagnóstico de câncer comprovado histologicamente \\
\hline $\mathbf{0}$ & Necessidade de avaliação adicional (outras incidências \\
\hline
\end{tabular}

Fonte: Adaptado do ACR (2016).

Esse sistema utiliza categorias de zero a seis na descrição dos achados dos exames radiológicos e prevê recomendação de conduta para cada categoria, com o objetivo de minimizar as diferenças de condutas inerentes à variabilidade ou discordância dos observadores. (Brasil, 2013)

Ao serem aplicados testes estatísticos revelou-se que houve evidência suficiente ao nível de significância de 5\% de que há uma associação entre o resultado das mamografias rastreio e diagnósticas e laudos categorizados pelo BI-RADS.

Pela análise descritiva houve maior prevalência de mamografias diagnóstico laudadas na categoria zero (30\%), em relação as de rastreio (16\%). Um estudo similar exibiu que $23 \%$ das mulheres que realizaram mamografia de rastreio não apresentavam nenhum tipo de achado. (Silva, Katz, Amorim, 2014)

Com a junção das categorias 1, 2 e 3, compara-se que o grupo da mamografia de rastreio apresentou quantidade superior (83\%) em relação as mamografias de diagnóstico (61\%) (Figura 2 e 3). Há resultado similar na literatura (16). Concomitante a isso, o agrupamento 4, 5 e 6, obteve maior prevalência no grupo diagnóstico (9\%), quando comparado ao grupo rastreio (1\%). Este resultado é análogo ao já apontado na literatura. Que leva a inferir que houveram mais achados de malignidade no grupo de mamografia diagnóstica. 
Figura 2. Resultado em valores absolutos do quantitativo de mamografias diagnóstico e rastreio laudadas, conforme classificação Bi-RADS, no estado do Tocantins (2015-2019).

\begin{tabular}{|c|c|c|c|c|}
\hline $\begin{array}{c}\text { Indicaçĩo } \\
\text { clinica }\end{array}$ & $\begin{array}{c}\text { Categoria } \\
\mathbf{0}\end{array}$ & $\begin{array}{c}\text { Categorias } \\
\mathbf{1 , 2} \mathbf{~ \mathbf { 3 }}\end{array}$ & $\begin{array}{c}\text { Categorias } \\
\mathbf{4 , 5} \mathbf{5} \mathbf{6}\end{array}$ & $\mathbf{P}$ \\
\hline $\begin{array}{c}\text { Mamografia } \\
\text { Diagnóstica }\end{array}$ & 218 & 440 & 69 & 0,005 \\
\hline $\begin{array}{c}\text { Mamografia } \\
\text { de Rastreio }\end{array}$ & 8222 & 42615 & 460 & 0,002 E-30 \\
\hline
\end{tabular}

Fonte: DATA SUS Ministério da Saúde.

Figura 3. Resultado percentual de mamografias diagnóstico e rastreio laudadas, conforme classificação BI-RADS, no estado do Tocantins (2015-2019).

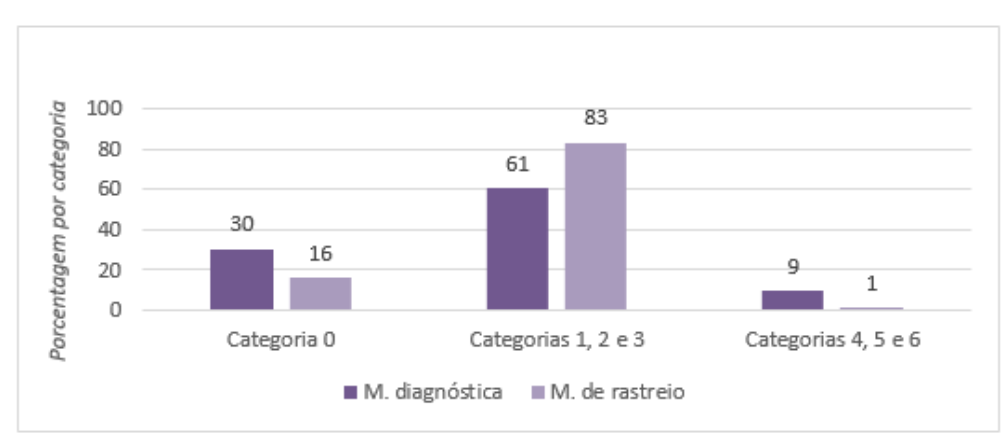

Fonte: DATA SUS Ministério da Saúde.

Além disso, também por testes estatísticos revelou-se que houve evidência suficiente ao mesmo nível de significância anterior, de que há uma associação entre pacientes por laudo de mamografias de rastreio e diagnósticos e faixa etária das pacientes (Figura 4 e 5).

A análise descritiva revela que houve maior prevalência de achados de suspeita de malignidade a diagnóstico de câncer (categorias 4, 5 e 6) aos 40 a 64 anos (78,6\%), em relação a faixa etária de 65 a acima de 79 anos (14,2\%) e a faixa etária de 15 a 39 anos $(7,2 \%)$. 
Figura 4. Resultado em valores absolutos do quantitativo de mamografias diagnóstico e rastreio laudadas, conforme classificação Bi-RADS, categorias 4, 5 e 6, e faixa etária, no estado do Tocantins (2015-2019).

\begin{tabular}{|c|c|c|}
\hline Faixa etária & Indicação Clínica & Categorias 4,5 e 6 \\
\hline $\begin{array}{c}\text { Entre } 15 \\
\text { a } 39 \text { anos }\end{array}$ & Mamografia Diagnóstica & 10 \\
\cline { 2 - 3 } & Mamografia de Rastreio & 27 \\
\hline $\begin{array}{c}\text { Entre } 40 \\
\text { a } 64 \text { anos }\end{array}$ & Mamografia Diagnóstica & 48 \\
\hline Entre 65 & Mamografia de Rastreio & 356 \\
\hline acima de 79 anos & Mamografia de Rastreio & 10 \\
\hline
\end{tabular}

Fonte: DATA SUS Ministério da Saúde.

Figura 5. Resultado percentual das categorias 4, 5 e 6, segundo a classificação BI-RADS, conforme faixa etária, no estado do Tocantins (2015-2019).

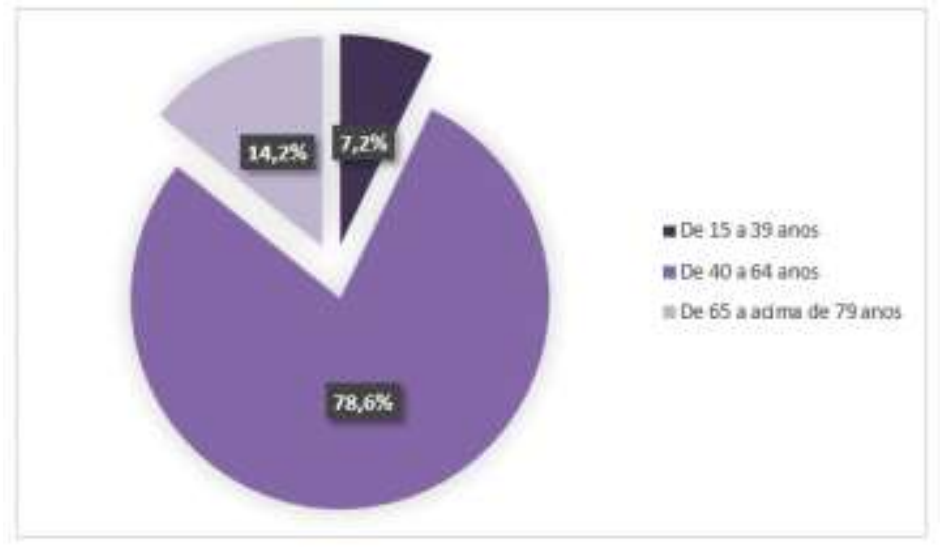

Fonte: DATA SUS Ministério da Saúde.

Também através de análises estatísticas foi revelado que houve evidência suficiente ao mesmo nível de significância de $5 \%$, de que há uma associação entre pacientes por laudo de mamografias de rastreio e diagnósticos e o risco elevado (Figura 6 e 7).

A análise descritiva revela que houve maior prevalência de pacientes que não tem risco elevado (63,6\%), em vista de pacientes que não sabem $(21,7 \%)$, pacientes que tem risco elevado $(14,4 \%)$ e com risco elevado ignorado $(0,3 \%)$. 
Figura 6. Resultado em valores absolutos do quantitativo de mamografias diagnóstico e rastreio laudadas, conforme classificação Bi-RADS, e o risco elevado, no estado do Tocantins (2015-2019).

\begin{tabular}{|c|c|c|c|c|}
\hline Risco elevado & Indicação Clínica & Categorias 0 & Categorias 1, 2 e 3 & Categorias 4, 5 e 6 \\
\hline \multirow{2}{*}{ Sim } & Mamografia Diagnóstica & 45 & 97 & 27 \\
\cline { 2 - 5 } & Mamografia de Rastreio & 1238 & 5544 & 103 \\
\hline \multirow{2}{*}{ Não } & Mamografia Diagnóstica & 130 & 259 & 38 \\
\cline { 2 - 5 } & Mamografia de Rastreio & 5020 & 25544 & 240 \\
\hline \multirow{2}{*}{ Não Sabe } & Mamografia Diagnóstica & 33 & 61 & 3 \\
\cline { 2 - 5 } & Mamografia de Rastreio & 1553 & 8883 & 102 \\
\hline \multirow{2}{*}{ Ignorado } & Mamografia Diagnóstica & 7 & 23 & 0 \\
\cline { 2 - 5 } & Mamografia de Rastreio & 21 & 114 & 2 \\
\hline
\end{tabular}

Fonte: DATA SUS Ministério da Saúde.

Figura 7. Resultado percentual segundo a classificação BI-RADS, conforme risco elevado, no estado do Tocantins (20152019).

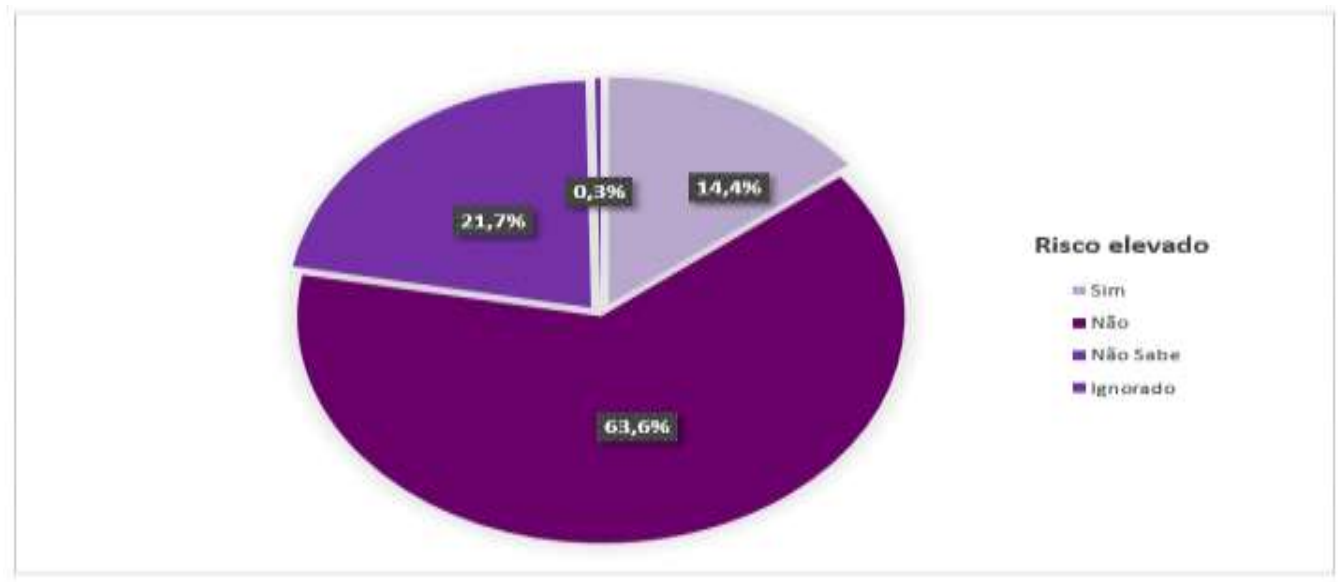

Fonte: DATA SUS Ministério da Saúde.

\section{Conclusão}

A partir do presente estudo pode-se perceber o quanto a mamografia de rastreio é importante na identificação de alterações (benignas e malignas). Houve maior prevalência de preditivos de malignidade na mamografia de diagnóstico. Foi possível concluir que a faixa etária de 40 a 64 anos apresentou maior porcentagem de possibilidade de achados em Bi-Rads. Já na relação entre a existência de risco elevado e a classificação Bi-Rads, houve uma maior porcentagem de possiblidades de achados nas mulheres que não informaram preexistência de fator de risco na consulta. Deve-se ressaltar que são necessárias ações de rastreamento e diagnóstico precoce conforme as recomendações do Ministério da Saúde, bem como aperfeiçoamento do sistema de laudos, para não haver comprometimento de efetividade do rastreio de câncer de mama.

É imprescindível que outras pesquisas no campo da detecção do câncer de mama sejam realizadas, como também grupos de discussões sobre a importância da temática, pois somente assim conseguiremos aumentar o numero de acesso a informação quanto ao diagnóstico rápido de um câncer de mama. 


\section{Referências}

American Cancer Society. Cancer facts \& figures 2019. American Cancer Society; 2019.

ACR. (2016). Atlas BIRADS® do ACR: sistema de laudos e registro de dados de imagem da mama. (5a ed.), Tradução de Angela Caracik. American College Radiology (ACR). Colégio Brasileiro de Radiologia e Diagnóstico por Imagem.

Brasil. (2013). Sistema de informação do câncer: manual preliminar para apoio à implantação. Ministério da Saúde. Instituto Nacional De Câncer José Alencar Gomes Da Silva. INCA.

Brasil. (2019). A situação do câncer de mama no Brasil: síntese de dados dos sistemas de informação Ministério da Saúde. Instituto Nacional De Câncer José Alencar Gomes Da Silva. INCA.

Brasil. (2015) Diretrizes para a detecção precoce do câncer de mama no Brasil. Ministério da Saúde. Instituto Nacional De Câncer José Alencar Gomes Da Silva. INCA.

Brasil. (2019) Estimativa 2020: incidência de câncer no Brasil. Ministério da Saúde. Instituto Nacional De Câncer José Alencar Gomes Da Silva. INCA.

Brasil. (2013). Institui a Política Nacional para a Prevenção e Controle do Câncer na Rede de Atenção à Saúde Ministério da Saúde. Gabinete do Ministro.Portaria nº 874/GM, de 16 maio 2013. Diário Oficial da União. Diário Oficial da União, p.129- 132, Seção 1.

Brasil. (2006). Controle dos cânceres do colo do útero e da mama Secretaria de Atenção à Saúde. Departamento de Atenção Básica. Controle dos cânceres do colo do útero e da mama. Brasília: Ministério da Saúde.

Ferlay, J., Soerjomataram, I., Dikshit, R., Eser, S., Mathers, C., Rebelo, M., Parkin, D. M., Forman, D., \& Bray, F. Cancerincidenceandmortalityworldwide: sources, methodsand major patterns in GLOBOCAN 2012. Int J Cancer. 136(5):E359-86.

Godinho, E. R. \& Koch, H. A. (2002). O perfil da mulher que se submete a mamografia em Goiânia: uma contribuição a bases para um programa de detecção precoce do câncer de mama. Radiol Bras. 35(3): 139-45.

Kemp C, Petti D. A., Ferraro O., \& Elias S. (2002). Câncer de mama: prevenção secundária. Sociedade Brasileira de Mastologia, Federação Brasileira das Sociedades de Ginecologia e Obstetrícia. Projeto Diretrizes. Associação Médica Brasileira, Conselho Federal de Medicina.

Nascimento F. B., Pitta M. G. da R. \& Rêgo M. J. B. M. (2015) Análise dos principais métodos de diagnóstico de câncer de mama como propulsores no processo inovativo. ArqMed 29(6): 153-159.

Mendes, K.D. S.; Silveira, R. C. de C. P \& Galvão, C. M. (2008). Revisão Integrativa: Método de pesquisa para a incorporação de evidências na saúde e na enfermagem. Texto \& Contexto Enfermagem, 17(4), 758-64.

MigowskiArn, S. A. T., et al. (2018) Diretrizes para detecção precoce do câncer de mama no Brasil. I - Métodos de elaboração. Cad. Saúde Pública 34(6): e00116317.

MigowskiArn, S. G. et al (2018). Diretrizes para detecção precoce do câncer de mama no Brasil. II - Novas recomendações nacionais, principais evidências e controvérsias. Cad. Saúde Pública 34(6):e00074817.

Minayo MCS. (1999) O desafio do conhecimento: pesquisa qualitativa em Saúde. HUCITEC, ABRASCO.

Rodrigues, D. C. N. et al (2013) Performance ofdiagnostic centers in theclassificationofopportunisticscreeningmammogramsfromtheBrazilianpublichealth system (SUS). RadiolBras 46(3): 149-155.

Sacket, d \& L. et al. (2003) Medicina baseada em evidências: prática e ensino. (2a ed.), Artmed.

Silva, F. X., et al (2014). Mamografia em mulheres assintomáticas na faixa etária de 40 a 49 anos. Rev. Saúde Pública (6): 931-939.

Tobar, F., \& Yalour, M. R. (2001) Como fazer teses em saúde publica: conselhos e idéias para formular projetos e redigir teses e informes de pesquisa. Editora Fiocruz.

World Cancer Research Fund. (2018) American Institute For Cancer Research. Diet, nutrition, physical activity and cancer:a global perspective. Continuous Update Project Expert Report.

World Health Organization. (2007) Cancercontrol. Knowledgeintoaction: WHO guide for effectiveprogrammes. Earlydetection; module 3. World Health Organization 